\title{
Evaluation Schema for SAR image Segmentation based on Swarm Optimization in Neutrosophic Domain
}

\author{
Mangalraj.P \\ IIIT-Allahabad \\ mangal86@gmail.com
}

\author{
Rakesh Singala \\ MANIT-Bhopal \\ rakeshsingalanitbpl@gmail.com
}

\author{
Anupam Agrawal \\ IIIT-Allahabad \\ anupam69@gmail.com
}

\begin{abstract}
The present paper proposes an evaluation schema for $S A R$ image Segmentation. The segmentation of $S A R$ images becomes a crucial step because the segmented results will be used as the input for the post- processing like target detection, change detection applications. The preprocessing task segmentation is highly influenced by inherent noise known as speckle. The proposed system introduces the evaluation schema for segmentation of $S A R$ images in Neutrosophic domain, which overcome the problem of speckle. The system is evaluated for different Swarm Optimization algorithms and the promoting results are obtained through the proposed evaluation schema. The results are discussed on the basis of different parameters.
\end{abstract}

Key words - SAR images, Swarm Optimization, Neutrosophic domain

\section{INTRODUCTION}

Image segmentation is a process of extracting the region of interest from an image based on the property called homogeneity. The union of two adjacent regions is not homogeneous. Mathematically: - partition of Image I into Non-Overlapping regions $\mathrm{S}_{\mathrm{i}}$.

$$
\text { mathop } \bigcup S_{i}=I \text { and } S_{i} \cap S_{j}=\phi, \quad i \neq j
$$

Image segmentation has become one of the knotty problems in the image processing domain due to the intricacy and heterogeneity. The segmentation outcomes are usually regulated by factors like illuminating, contrast, noise, etc. Most of the segmentation techniques having its ground on two properties of pixels in their local neighborhood: the discontinuity and similarity. The approach based on discontinuity tends to partition an image by detecting isolated points, lines and edges according to abrupt changes in intensities [5]. Impediment in segmentation due to several inevitable factors such as intensity variations in image and noises. The efficiency of segmentation techniques should be figure out through its robustness and consistency abilities.

Synthetic aperture radar (SAR) an active imagery system which produces high resolution images which is having major applications in the fields of Military, Agriculture and Urban development. Image segmentation is an evidential part of SAR image analysis task. Segmentation of SAR images is bifurcated into two categories: feature-based algorithms and model-based algorithms
Feature-based algorithms are highly sensitive to Speckles, an inherent noise which is present in SAR images due to the coherent imaging. Due to speckles the yielded images through feature based algorithms are highly corrupted. To avoid this situation a robust filtering technique is needed as a former step of segmentation. Model-based methods having high computational complexity which gives major impacts on real time system, model-based methods do not solve the issue of disconnected region segmentation and poor contrast in the SAR images [1-4]. Due to the above mentioned problems like speckle hindrance, computational complexity the area is still open for SAR imagery data which encourages a new segmentation system and evaluations.

Fuzzy based system for image segmentation and preceded the fuzziness of image pixels lead to the success of these systems. The uncertainties and inaccuracies are basically dealt with fuzzy systems [6]. Major recede of the fuzzy systems are due to the hindrances of the speckles or noises present in the image. To handle uncertainty and indeterminate values a neutral based system called as the Neutrosophic based systems has utilized for our evaluation schema. The effectiveness of our system is evaluated based on some parameters which we discussed in the experimental results and discussion section. The evaluations showed that our method produces and output which have the ability to overcome the above mentioned problems like speckle influence, false edges, Low contrast and illumination effects. The system has been checked for its robustness and computational complexity and at the same time for consistency is also discussed in the experimental results and discussion section.

\section{NEUTROSOPHIC DOMAIN [6], [7]}

Neutrosophic logic (NL) is a blend of different logics like fuzzy, Para consistent and intuitionistic logic. The basic theme behind the NL is the representation of data in a 3D space in which dimensions represents truth $(T)$, false $(F)$ and Indeterminacy (I), where T, F and I are subsets of

]$^{-} 0,1^{+}[$may or may not be related (Non-standard unit interval). 


\section{Neutrosophic Sets}

Let $U$ be a universal set in which $M$ is a subset of $U$. An element ' $\mathrm{x}$ ' belong to $\mathrm{M}$ which can be represented as $\mathrm{x}(\mathrm{T}, \mathrm{F}, \mathrm{I})$ means its $\mathrm{t} \%$ true in set, $\mathrm{f} \%$ false in set and $\mathrm{i} \%$ indeterminate in set where $t$ varies in $T$, $f$ varies at $F$ and $i$ varies at $I$.

Basically ( $\mathrm{T}, \mathrm{F}$ and I) are subsets but in depth these are the functions or operators for transformations. Neutrosophic sets are derived based on the neutrality which is one of the generalized forms of intuitionistic fuzzy sets for solving indeterminacy problem.

\section{Neutrosophic sets and Image Processing}

Let $T, I, F$ be real standard subset of $]^{-} 0,1^{+}[$with

$\sup \mathrm{T}=\mathrm{t} \_$sup, $\inf \mathrm{T}=\mathrm{t} \_$inf,

$\sup I=i \_$sup, $\inf I=i \_$inf,

$\sup F=f_{-} \sup , \inf F=f_{-} \inf$,

where sup and inf are the supremum and an infimum properties for a real number system.

$$
\text { n_sup }=\mathrm{t} \_ \text {sup }+\mathrm{i} \_ \text {sup }+\mathrm{f} \_ \text {sup; }
$$$$
\mathrm{n} \_ \text {inf }=\mathrm{t} \_ \text {inf }+\mathrm{i} \_ \text {inf }+\mathrm{f} \_ \text {inf; }
$$

Here (T, I and F) represents the Neutrosophic Components. The Neutrosophic set is imposed on image processing in the following way $I M \subseteq U$ where IM is the image which is composed with bright pixels and pixel is described as $\mathrm{p}(\mathrm{t}, \mathrm{i}, \mathrm{f})$ and belongs to IM in the following way $\mathrm{t} \%$ true in set, $\mathrm{f} \%$ false in set and $\mathrm{i} \%$ indeterminate in set where $t$ varies in $T$, $f$ varies at $F$ and $i$ varies at $I$.

Pixel Transform from Image Domain to Neutrosophic Domain [8]

$$
P_{i m}(\mathrm{i}, \mathrm{j})=P_{N S}(\mathrm{i}, \mathrm{j})=\{\mathrm{T}(\mathrm{i}, \mathrm{j}), \mathrm{I}(\mathrm{i}, \mathrm{j}), \mathrm{F}(\mathrm{i}, \mathrm{j})\}
$$

Where $T(i, j), F(i, j)$ and $I(i, j)$ are defined as the membership values which can be derived as follows

$$
T(\mathrm{i}, \mathrm{j})=\frac{\bar{g}(\mathrm{i}, \mathrm{j})-\bar{g}(\min )}{\bar{g}(\max )-\bar{g}(\min )}
$$

Where $g(i, j)$ is the Intensity value of pixel at location $i, j$ $\bar{g}(\mathrm{i}, \mathrm{j})$ is the mean local value and it can be derived as follows

$$
\bar{g}(\mathrm{i}, \mathrm{j})=\frac{1}{M X M} \sum_{m=i-M / 2}^{i+M / 2} \sum_{n=i-M / 2}^{i+M / 2} g(\mathrm{~m}, \mathrm{n})
$$

MxM denotes the window size.

$$
F(i, j)=1-T(i, j)
$$

$$
\begin{aligned}
& \mathrm{I}(\mathrm{i}, \mathrm{j})=\frac{\delta(\mathrm{i}, \mathrm{j})-\delta(\min )}{\delta(\max )-\delta(\min )} \\
& \delta(\mathrm{i}, \mathrm{j})=\operatorname{abs}(\mathrm{g}(\mathrm{i}, \mathrm{j})-\bar{g}(\mathrm{i}, \mathrm{j})
\end{aligned}
$$

The value of $I(i, j)$ is utilized to evaluate the indeterminacy degree of element $P_{N S}(\mathrm{i}, \mathrm{j})$ and $\mathrm{T}$ and $\mathrm{F}$ are correlated with I, and influenced in pixel distribution of I and entropy of I.

$\alpha$ - Mean and $\beta$ - Enhancement utilized to decrease the set indeterminacy on NS domain and achieved as follows

Image mean is derived as follows

$$
\begin{aligned}
& \bar{I}(\mathrm{i}, \mathrm{j})=\frac{1}{M X M} \sum_{m=i-M / 2}^{i+M / 2} \sum_{n=i-M / 2}^{i+M / 2} \mathrm{I}(\mathrm{m}, \mathrm{n}) \\
& \alpha \text { - Mean operation } P_{N S}^{-}(\alpha) \text { is desired } \\
& P_{N S}^{-}(\alpha)=\bar{P}(T(\alpha), \overline{\mathrm{I}}(\alpha), \overline{\mathrm{F}}(\alpha))
\end{aligned}
$$

Where

$$
\begin{aligned}
& \vec{T}(\alpha)=\left\{\begin{array}{l}
T, 1<\alpha \\
\bar{T}, I \geq \alpha
\end{array}\right\} \\
& \bar{T}(\mathrm{i}, \mathrm{j})=\frac{1}{M X M} \sum_{m=i-M / 2}^{i+M / 2} \sum_{n=i-M / 2}^{i+M / 2} \mathrm{~T}(\mathrm{~m}, \mathrm{n}) \\
& \bar{F}(\alpha)=\left\{\begin{array}{l}
\mathrm{F}, \mathrm{I}<\alpha \\
\bar{F}, I \geq \alpha
\end{array}\right\}
\end{aligned}
$$

$$
\bar{F}(\mathrm{i}, \mathrm{j})=\frac{1}{M X M} \sum_{m=i-M / 2}^{i+M / 2} \sum_{n=i-M / 2}^{i+M / 2} \mathrm{~F}(\mathrm{~m}, \mathrm{n})
$$

$$
I_{\alpha}(\mathrm{i}, \mathrm{j})=\frac{\bar{\delta}(\mathrm{i}, \mathrm{j})-\bar{\delta}(\min )}{\bar{\delta}(\max )-\bar{\delta}(\min )}
$$

$$
\bar{\delta}(\mathrm{i}, \mathrm{j})=\operatorname{abs}(\bar{T}(\mathrm{i}, \mathrm{j})-\bar{T}(\mathrm{i}, \mathrm{j}))
$$

$$
\bar{T}(\mathrm{i}, \mathrm{j})=\frac{1}{M X M} \sum_{m=i-M / 2}^{i+M / 2} \sum_{n=i-M / 2}^{i+M / 2} \bar{T}(\mathrm{~m}, \mathrm{n})
$$


After $\alpha$ operation the entropy of the indeterminate subset I is increased consequently IM has more uniform element distribution [9], [10], [11].

$\mathrm{F}$ set we will neglect for further processing now we choose only $\mathrm{I}$ and $\mathrm{T}$ i.e. True and indeterminate set only. Create a co-occurrence matrix by using the sets I and T which will provide fitness value for the optimization algorithms [12]. We are not interested with $\beta$ Enhancement because already our input image is $\log$ transformed i.e. already the input SAR image contrast is enhanced by log transform.

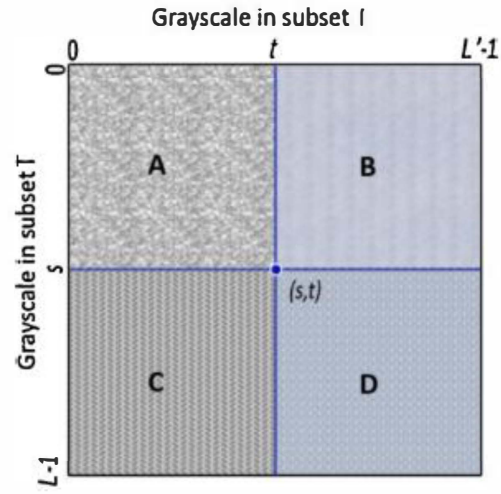

Fig. 1. Co-occurrence Matrix

Two dimensional threshold vector $(\mathrm{s}, \mathrm{t})$ can portion cooccurrence matrix in 4 quadrants A,B,C,D as shown in fig in which $\mathrm{A}$ and $\mathrm{D}$ are present object and background respectively $\mathrm{B}$ represents the edges and texture feature in background regions. $\mathrm{C}$ having same values as A they are used in entropy calculation. If Entropy change is small the intensity distribution is Non-Uniform.

\section{SWARM OPTIMIZATION ALGORITHMS}

Swarm Intelligence designs an efficient computational method for solving problems in a fashion which is inspired through the behavior of real swarms. Different models have been designed so far on the basis of swarm's peculiar behavior. Cardinally all the swarms are having the basic principles: Self-Organization and collective responses. Some of the swarm optimizations such as Particle Swarm Optimization, Ant colony Optimization and Bee Colony optimization and their promising features lead to a strong foundation of developing models for solving problems in an intelligent way.

\section{Particle Swarm Optimization [15], [16]}

Particle swarm optimization (PSO) is a population-based algorithm based on the social behavior of birds in a flock. PSO is initialized with a random generation of population and then searches for optima by updating generations. Each particle is flown through the search space having its velocity by their local optimum made impacts over the global optimum. The performance of each particle is based on the proximity of their optimum against the global one, which usually defined through the problem.

\section{Artificial Bee Colony [13], [14]}

Artificial Bee Colony (ABC) is a population-based algorithm grounded on the intelligent behaviors of real bees Foraging is the basic behavior of bee for that purpose 3 types of bees are used namely employed, onlooker and scout bees .All the employed bee search the food and update it on regular intervals that means the total no of food source equal to the number of employed bees. Next step is that food sources are selected by the onlooker bees probabilistically. Employed bee whose food source is abandoned becomes a scout. The area of food sources is relevant with the possible solutions of optimization problems and the value of food sources is relevant with the value of problem solutions.

\section{Ant Colony Optimization [17], [18]}

Ant Colony Optimization (ACO) is also a populationbased algorithm grounded on the behavior of real ants. Ants will find an optimal path if the path gets breaks it will be generate the next optimal path by mutual transfer of information and substance called pheromone. Every ant will release pheromone in their own path and perceive its intensity for its own guidance. Ants will follow the path where pheromone intensity is high will become the optimal path. This scenario is mentioned as positive feedback process.

\section{PROPOSED METHODOLOGY - EVALUATION SCHEMA}

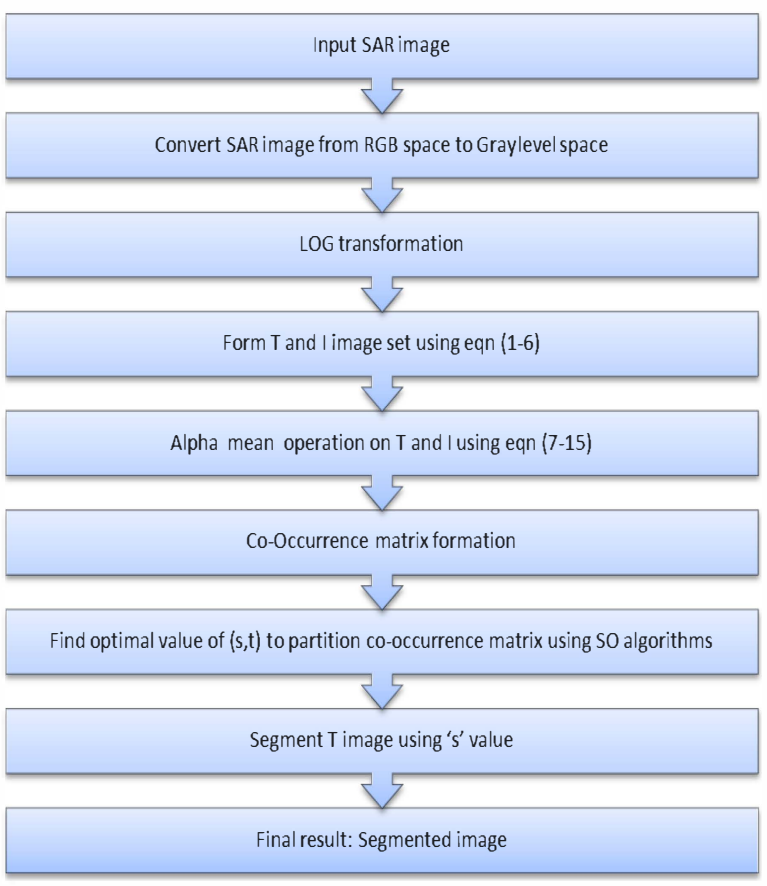

Fig. 2. Proposed Methodology 
The fig.2 depicts the proposed methodology for an Evaluation schema for SAR segmentation system. In the system the optimal value to partition the co-occurrence matrix is derived from the Swarm Optimization algorithms. Further Partitioned $\mathrm{T}$ image is used for segmentation using the optimal value. The later section will give the comprehensive evaluations of the Swarm Optimization algorithms in Neutrosophic Domain.

\section{EXPERIMENTAL RESULTS AND DISCUSSION}

In this section, synthetic and real SAR images are utilized to illustrate the performance of the proposed algorithm. The system has evaluated for Swarm Optimization Techniques for SAR image segmentation. In this experiment, for SO algorithms, the population size is 20 , the number of iterations is 30 , and the limit times for abandonment is 10 , the lower and upper bounds are 0 and 255 respectively.

Basic conditions

1. Total run $=3$ runs

2. Total cycle $=30 /$ run

3. Stopping criteria $=$ same optimal value repeats.

4. In case the stopping criteria is not fulfilled the number of cycles per run will be increased to 60 .

Two Data Sets have been used for our Evaluation Purpose.

A. Pipeline over the Rio Grande river near Albuquerque, New Mexico - Air Craft Based SAR imagery, captured band is ku with a resolution of 1$\mathrm{m}$.

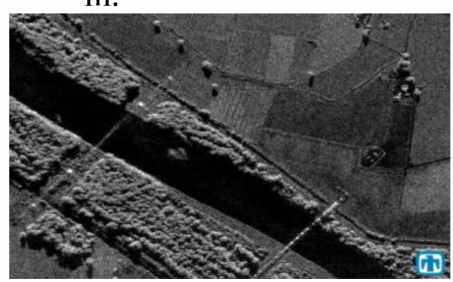

Fig.3. Original Pipeline Dataset

B. Subset 2 RS2-SLC-FQ15-DES-06-May-

2008_14_Intensity_HH:- Radarsat 2 Data of Vancouver region, Canada - Single Look complex Data having a resolution of 3 meters and for our purpose horizontal Polarized Intensity image is used.

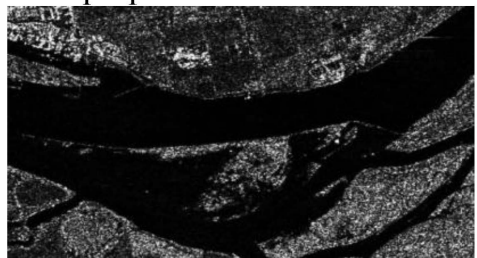

Fig.4. Original Radarsat2 Dataset.

\section{5. a. RESULTS}

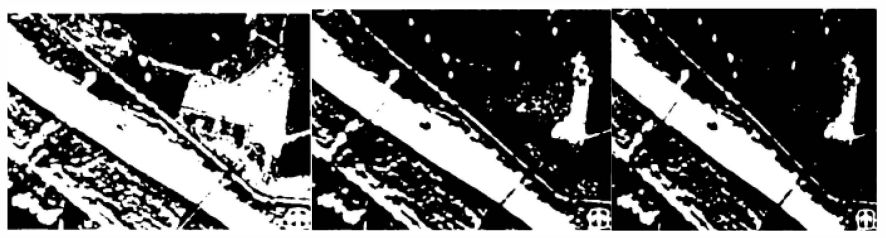

a. PSO

b. ACO

c. $A B C$

Fig.5. Visual Segmented results for Data set.1.

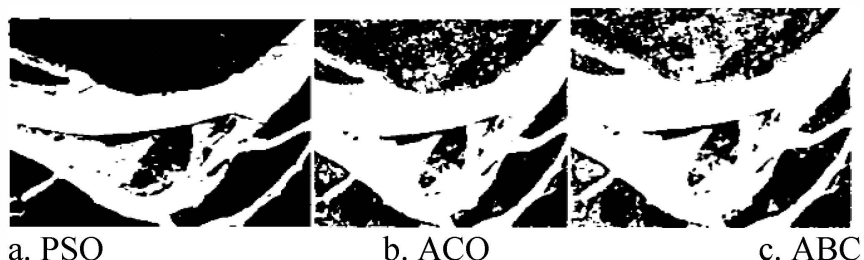

Fig.6. Visual Segmented results for Data set.2.

Table 1. Results on SSIM parameter

\begin{tabular}{|l|l|l|l|}
\hline Data set & PSO & ACO & ABC \\
\hline$[\mathrm{A}]$ & .7496 & .7233 & .6683 \\
\hline$[\mathrm{B}]$ & .777 & .7459 & .7218 \\
\hline
\end{tabular}

Table 2. Results on ENL parameter

\begin{tabular}{|l|l|l|l|}
\hline Data set & PSO & ACO & ABC \\
\hline$[\mathrm{A}]$ & 1.0399 & 1.1259 & 1.495 \\
\hline$[\mathrm{B}]$ & .904 & .946 & 1.14 \\
\hline
\end{tabular}

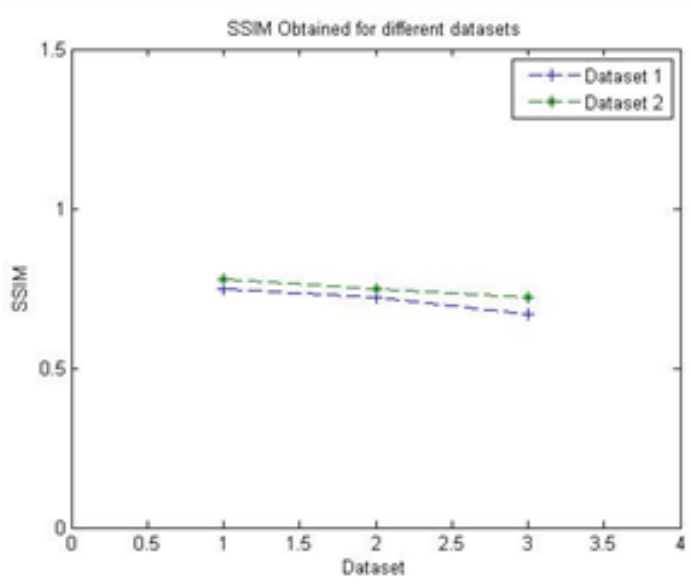

Fig.7. SSIM comparison 


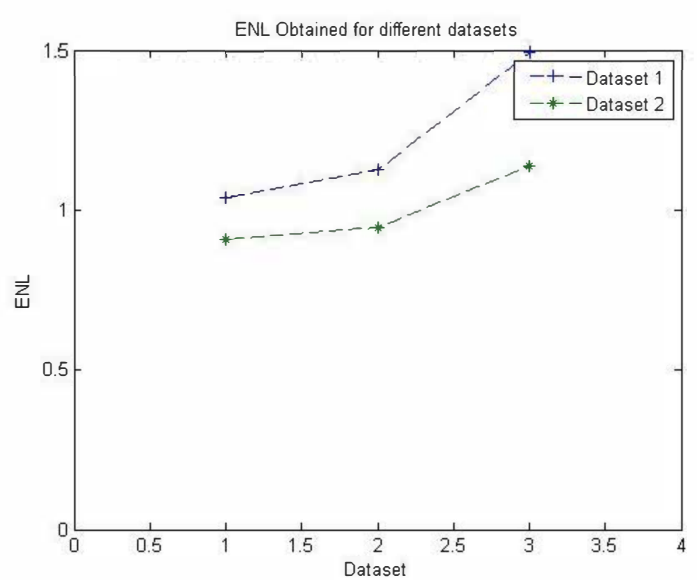

Fig.8. ENL comparison

\section{5. b. DISCUSSION}

The parameters SSIM and ENL are used for the qualitative measures over the segmentation results. The SSIM parameter value should be low for good segmentation results and the ENL parameter value should be high for good segmentation results. By considering the results on table 1 and table 2 the evaluation results show that the $\mathrm{ABC}$ algorithm in Neutrosophic domain produces very good segmentation results. The fig 5 and fig 6 shows the segmented visual results over the data sets and $\mathrm{ABC}$ provides more details in the segmented results if we visualize the results more carefully PSO visual results are not so good when compared to $\mathrm{ACO}$ and $\mathrm{ABC}$ in our proposed system.

Fig 7 and Fig 8 shows the comparison graph of SSIM and ENL results on different dataset and it is noticed that the results of $\mathrm{ABC}$ algorithm on both the datasets a better result than the other optimization algorithms. The evaluation schema has been tested against some the bench mark data sets [A] and real time data set [B] and in the datasets the proposed schema is able to produce a boosting results.

\section{CONCLUSION}

The presented paper proposed an evaluation schema for SAR imagery segmentation in Neutrosophic domain using the SO techniques which obtains clear object boundaries. The proposed evaluation system has reduced the influence of speckle during the segmentation process. This evaluation schema is evaluated for $3 \mathrm{SO}$ algorithms and provided distinct results over different datasets. It is concluded from the discussion that $\mathrm{ABC}$ algorithm provides better promoting results against the other two $\mathrm{SO}$ algorithms in the evaluation system.

\section{ACKNOWLEDGEMENT}

The authors would like to express their deep gratitude towards Mr. Manjunath Bhandary, Founder, Bhandary
Foundation - Mangalore, for the constant support of this research work.

\section{REFERENCES}

[1]. Lee, Jong-Sen and Igor Jurkevich, "Segmentation of SAR images," IEEE transactions on Geoscience and Remote Sensing, vol.27, issue.6, pp. 674-680, 1989.

[2]. El Zaart, Ziou D, Wang S, and Jiang Q, "Segmentation of SAR images," Elsevier Pattern Recognition, vol.35, No.3, pp 713-724, 2002.

[3]. Touzi, Ridha, Armand Lopes, and Pierre Bousquet "A statistical and geometrical edge detector for SAR images," IEEE Transactions on Geoscience and Remote Sensing, Vol.26, no. 6, pp. 764-773, 1988.

[4]. Robert O Harger, Synthetic Aperture Radar Systems Theory and Design, New York, Academic Press, 1970.

[5]. Ming Zhang, "Novel Approaches to Image Segmentation Based on Neutrosophic Domain," PhD. thesis, computer science, utah state university, Logan, Utah, 2010.

[6]. Neutrosophy, Neutrosophic Logic, Neutrosophic Set, Neutrosophic Probability and Statistics, http://www.gallup.unm.edu/ smarandache/neutrosophy.ht m (Accessed: 28-August-2014).

[7]. Rivieccio Umberto, "Neutrosophic logics: Prospects and problems," Elsevier Fuzzy Sets and Systems, vol. 159, no. 14, pp. 1860-1868, 2008.

[8]. Yanhui Guo and H.D. Cheng, "New neutrosophic approach to image segmentation." Elsevier Pattern Recognition, vol. 42, no. 5, pp. 587 - 595, 2009.

[9]. Yanhui Guo and Abdulkadir Şengür, "A novel image edge detection algorithm based on neutrosophic set," Elsevier Computers and Electrical Engineering, 2014.

[10]. Zhang Ming, Ling Zhang and H. D. Cheng, "A neutrosophic approach to image segmentation based on watershed method," Elsevier Signal Processing, vol. 90, no. 5 , pp. $1510-1517,2010$.

[11]. Abdulkadir sengur, and Yanhui Guo. "Color texture image segmentation based on neutrosophic set and wavelet transformation," Elsevier Computer Vision and Image Understanding, vol. 115, no. 8, pp. 1134-1144, 2011.

[12]. Kazim Hanbay, and M. Fatih Talu, "Segmentation of SAR images using improved artificial bee colony algorithm and neutrosophic set," Elsevier Applied Soft Computing, vol. 21, pp. 433-443, 2014.

[13].D. Karaboga and B. Basturk, "On the performance of artificial bee colony $(\mathrm{ABC})$ algorithm," Elsevier Applied Soft Computing, vol.8, pp.687-697, 2008.

[14]. Karaboga Dervis, "An idea based on honey bee swarm for numerical optimization," Vol. 200. Technical report-tr06, Erciyes University, Engineering faculty, computer engineering department, 2005.

[15]. James Kennedy, "Particle swarm optimization." Springer In Encyclopedia of Machine Learning, pp. 760-766, 2010.

[16].Ioan Cristian Trelea, "The particle swarm optimization algorithm: convergence analysis and parameter selection," Elsevier Information processing letters, vol. 85, no. 6, pp. 317-325, 2003. 
[17]. Marco Dorigo and Christian Blum, "Ant colony optimization theory: A survey," Elsevier Theoretical computer science, vol. 344, no. 2, pp.243-278, 2005.

[18]. Marco Dorigo and Mauro Birattari, "Ant colony optimization," Springer In Encyclopedia of Machine Learning, $\quad$ pp. 36-39, 2010. 